\title{
Evaluation des primären Rehabilitationserfolgs in der wohnortnahen dermatologischen Rehabilitation bei Psoriasis-Patienten
}

\section{Kardorff1,2,3}

\author{
Evaluation of Primary Rehabilitation Outcome in a Neighbourhood \\ Rehabilitation Program for Psoriasis Patients
}

\section{Zusammenfassung}

Einleitung: Seit 1996 wird im Ruhrgebiet (Duisburg) das Modellkonzept der wohnortnahen dermatologischen Rehabilitation im städtischen Ballungsgebiet praktiziert. Für die nahe Zukunft ist eine flächendeckende Patientenversorgung analog dieses Modells vorgesehen. Die nachfolgende Arbeit evaluiert die primären ortsnahen Rehabilitationsergebnisse bei Psoriasis und vergleicht mit Ergebnissen der wohnortfernen Rehabilitation. Patienten und Methodik: Daten von 292 konsekutiv wohnortnah ambulant und stationär rehabilitierten Psoriasis-Patienten wurden retrospektiv anhand des PASI-Scores und eines anonymisierten Fragebogens ausgewertet. Ergebnisse: In durchschnittlich 26 Behandlungstagen wurde eine Verbesserung des PASI-Scores von 20,2 $\pm 11,2$ auf 4,5 \pm 4,6 Punkte, im Median um 82,2\% erzielt. $83,4 \%$ der Patienten beurteilten den wohnortnahen Kontakt zu ihren Angehörigen während der Rehabilitation als wichtig. 12,6\% hätten eine wohnortferne Behandlung vorgezogen. Bei gleich guten Behandlungserfolgen erwies sich die stationäre wohnortnahe Rehabilitation um 65,5\% kostenintensiver als die ambulante. Schlussfolgerungen: Die wohnortnahe dermatologische Rehabilitation im Ruhrgebiet erbringt verglichen mit der traditionellen wohnortfernen Kur bei Psoriasis-Patienten vergleichbar gute primäre Rehabilitationserfolge. Die Nähe zum psychosozialen Umfeld ist dabei für die Patienten besonders wichtig. Bei gleich guten Behandlungsergebnissen ist die wohnortnahe ambulante Rehabilitation deutlich kostengünstiger als die stationäre.

\section{Abstract}

Introduction: Since February 1996 the model-concept for dermatological neighbourhood rehabilitation has been performed in the midst of an overcrowded urban area in Duisburg (Ruhrgebiet, Germany). For the near future care for patients is planned in centers like this in many areas of Germany. The primary evaluation results of the treatment of psoriasis in the concept „neighbourhood rehabilitation" are presented and compared to the results of rehabilitation centers far from home. Patients and Methods: Data of 292 consecutive in- and out-clinic psoriasis patients are retrospectively analysed concerning the PASI-scores and an anonymous questionnaire after discharge. Results: In an average of 26 treatment days an improvement of PASI-score from $20.2 \pm$ 11.2 to $4.5 \pm 4.6$ points, $82.2 \%$ in median could be achieved. $83.4 \%$ of the patients judged the contact to their family and friends during rehabilitation as important. $12.6 \%$ would have preferred a treatment far away from home. Achieving the same positive results the costs of neighbourhood in-patient rehabilitation were 65.5\% higher than the out-patient treatment. Conclusions: Dermatologic neighbourhood rehabilitation in the crowded RuhrArea shows similar positive primary rehabilitation results when compared to traditional spas far away from home. Especially being close to their psycho-socially environment is important for the patients in this context. While achieving similar positive results out-patients' neighbourhood rehabilitation causes distinctly less costs than in-patient rehabilitation.

Institutsangaben

${ }^{1}$ Rhein-Klinik St. Joseph, Rehabilitationszentrum für Dermatologie, Allergologie und Umweltmedizin

Duisburg (CA Dr. Johannes Kunze)

${ }^{2}$ Heinrich-Heine-Universität Düsseldorf Dermatologie (Direktor: Prof. Dr. Thomas Ruzicka)

${ }^{3}$ Gemeinschaftspraxis für Dermatologie, Allergologie, Phlebologie und Umweltmedizin,

Dres. B. Kardorff \& P. Dorittke, Mönchengladbach

Korrespondenzadresse

Maria Kardorff · Gemeinschaftspraxis Dr. med. Bernd Kardorff \& Dr. med. Peter Dorittke für Dermatologie, Allergologie, Phlebologie und Umweltmedizin · Marktstraße 31 . 41236 Mönchengladbach .

E-mail: kardorff@t-online.de 
Zusammen mit der durch den gemeinsamen Bundesausschuss beschlossenen Neufassung der Rehabilitationsrichtlinien [1] sind mit Datum vom 1.4.2004 die Rahmenempfehlungen zur ambulanten dermatologischen Rehabilitation [2] der Bundesarbeitsgemeinschaft für Rehabilitation Frankfurt a.M. (BAR) wirksam geworden. Damit rückt die Aussicht auf eine flächendeckende Versorgung chronisch hautkranker Menschen in wohnortnahen ambulanten dermatologischen Rehabilitationszentren in die nähere Zukunft. Aufgrund dieser aktuellen Ereignisse, stellt die nachfolgende Arbeit die Ergebnisse der Evaluation des primären Rehabilitationserfolgs von Patienten mit Psoriasis vulgaris vor, die in einem wohnortnahen dermatologischen Rehabilitationszentrum erzielt wurden, welches bereits seit der Eröffnung im Jahr 1996 nach den jetzt von der BAR [2] beschlossenen konzeptionellen, personellen, räumlichen, apparativen und therapeutischen Standards arbeitet [3-8]. Die nachfolgenden Daten geben zum einen Aufschluss über die Verbesserung des psoriatischen Hautbefunds während der wohnortnahen Rehabilitationsmaßnahme, zum anderen zeigen sie die hohe Patientenzufriedenheit mit einer Rehabilitation innerhalb des eigenen psychosozialen Umfelds. Abschließend werden die u.a. aufgrund unterschiedlicher Untersucher, unterschiedlicher Therapiekonzepte und differierender Behandlungsdauern lediglich tendenziell vergleichbaren Ergebnisse hinsichtlich der Besserung des Schweregrads des Hautbefunds anhand des PASI-Scores von traditionell wohnortfernen und wohnortnahen Einrichtungen nebeneinander gestellt.

\section{Patienten und Methodik}

\section{Patienten}

In die retrospektive Analyse wurden 292 konsekutive Patienten mit der Hauptdiagnose Psoriasis vulgaris (ICD-10: L40.0) aufgenommen, die in 3 aufeinanderfolgenden Jahren (1999-2001) wohnortnah in der Rhein-Klinik St. Joseph Duisburg rehabilitiert wurden. Nach der nach den aktuellen Rehabilitationsrichtlinien anzuwendenden internationalen Klassifikation der Funktionsfähigkeit, Behinderung und Gesundheit (ICF) [9] lagen bei den Patienten Schädigungen von Funktionen (ICF: b810-b869) und Strukturen der Haut- und Hautanhangsgebilde (ICF: s810-s899) z.B. in Form von Rötung, Schuppung, Rhagaden und Pusteln mit einhergehenden Schmerzen und Juckreiz vor. Im ICF-Bereich der Aktivitäten bestanden i.d.R. Beeinträchtigungen der persönlichen, sozialen, situationsbedingten und beruflichen Aktivitäten. Dadurch ist die Teilhabe in den Bereichen der körperlichen Unabhängigkeit (ICF: d510 ff.), der Mobilität (d410 ff.), der psychischen Belastbarkeit ( $d 240$ ), der sozialen Integration ( $d 710 \mathrm{ff}$.) und der Beschäftigung in Beruf (d840-d899) und Freizeit (d920) stark beeinträchtigt. 25 Patienten $(8,6 \%)$ litten zusätzlich an einer Psoriasisarthropathie (ICD-10: L40.5). Das Kollektiv von insgesamt 144 weiblichen und 148 männlichen Patienten zeigte folgende Altersverteilung: Alter zwischen 2 und 86 Jahren (Mittelwert 46,6 \pm 18,1, Median 47 Jahre). 205 Patienten (70,2\%) wurden ambulant und 87 Patienten $(29,8 \%)$ stationär rehabilitiert. Ambulante wie auch stationäre Patienten erhielten das identische medizinische, psychologische und physikalische Rehabilitationsprogramm über jeweils 6 Stunden täglich. Die Unterschiede be- standen werktags lediglich in der Übernachtung in der Klinik und entsprechend zusätzlicher Verpflegung bei den stationären Patienten und der häuslichen Übernachtung sowie der Gestaltung des Resttages im gewohnten psychosozialen Umfeld bei den ambulanten Patienten. An Samstagen, Sonn- und Feiertagen erhielten die stationären Patienten die gewohnte medizinische und physikalische Therapie. Lediglich das psychologische, soziale und Schulungs-Programm entfiel an den Wochenenden. Die Entscheidung darüber, ob bei einem Rehabilitanden eine stationäre oder ambulante Reha durchgeführt wurde, war abhängig vom Ausmaß der aufzuweisenden Schädigungen insgesamt (nicht vom Schweregrad des psoriatischen Hautbefalls), der Beeinträchtigung von Aktivitäten und Teilhabe, der räumlichen Entfernung vom Wohnort, der Verkehrsanbindung, vom sozialen Umfeld und berechtigten Wünschen des Rehabilitanden und entsprach bereits weitgehend den späteren Rahmenempfehlungen der BAR [2].

\section{Behandlungskonzept}

Das Therapiekonzept der Rhein-Klinik Duisburg [3-8] ist ganzheitlich ausgerichtet und verfolgt als übergeordnete Rehabilitationsziele u.a.

- die Abheilung bestehender psoriatischer Hautveränderungen und eine Verlängerung des hauterscheinungsfreien Intervalls,

- die Wiederherstellung und Erhaltung der Erwerbsfähigkeit,

- Hilfe bei der Findung von Bewältigungsstrategien und bei der Krankheitsverarbeitung zum erfolgreichen Langzeiterkrankungsmanagement,

- Beseitigung oder Minderung von Beeinträchtigungen im Familien- und Berufsleben, in der Freizeit und bei der Motivation,

- Abwendung drohender oder manifester Beeinträchtigungen der Teilhabe insbesondere in Bezug auf die physische Unabhängigkeit, die Mobilität, die psychische Stabilität, die soziale Integration und die Beschäftigung.

Zur Erreichung dieser Ziele kommen sowohl das komplette Spektrum dermatologischer Therapiemöglichkeiten (topische, systemische, Photo-Therapieverfahren) wie auch intensive psychotherapeutische Betreuung, physikalische und physiotherapeutische Therapien, ausgedehnte Patientenschulungen und Entspannungsverfahren zum Einsatz (Tab.1). Ermöglicht wird dieses breite therapeutische Spektrum durch ein interdisziplinäres Therapeutenteam. Die Tab. 1 zeigt die am häufigsten zum Einsatz gekommenen Therapieverfahren- und Kombinationsmodule, die aufgrund der individuellen Erstellung von Behandlungsplänen variieren. Ein Behandlungsschwerpunkt stellt, wie in anderen Kliniken auch (siehe Diskussion), die Balneophototherapie dar, sofern der Patient von seinem Alter und seiner Konstitution her dafür geeignet ist. Einen weiteren Therapieschwerpunkt sowie gleichzeitig ein Rehabilitationsziel stellt die Erlernung der Cignolin-Minutentherapie dar, die, gekonnt angewendet, für die $\mathrm{Pa}$ tienten eine langfristig wirksame und nebenwirkungsarme Therapieoption darstellt $[10,11]$. Intensive Anleitungen und Aufforderung zur konsequenten Hautpflege $[8,12]$ erhält jeder RehaPatient. Ebenso nimmt jeder Patient an einem psychologischen Aufnahmegespräch teil, infolgedessen sich die Notwendigkeiten zu weiteren Einzel- oder Gruppengesprächen ergeben. So weit sinnvoll, werden bei allen Therapiemodulen die familiären Bezugspersonen mit einbezogen, sowie im psychologischen Be- 
Tab. 1 Medizinische und psychotherapeutische Behandlungsmethoden während der wohnortnahen Rehabilitation

\begin{tabular}{|c|c|c|}
\hline $\begin{array}{l}\text { absolute Patientenzahl } \\
\text { (total, } n=292 \text { ) }\end{array}$ & $\begin{array}{l}{[\%]} \\
\text { der Patienten }\end{array}$ & $\begin{array}{l}\text { therapeutische } \\
\text { Kombinationsmodule }\end{array}$ \\
\hline 270 & 92,5 & Cignolin-Minutentherapie \\
\hline 216 & 74,0 & $\begin{array}{l}\text { Balneophototherapie } \\
\text { (15\%ige Starksole) }\end{array}$ \\
\hline 76 & 26,0 & Bade-PUVA-Therapie \\
\hline 153 & 52,4 & $\begin{array}{l}\text { topisches Calcipotriol } \\
{\text { (Daivonex }{ }^{\circledR}, \text { Psorcutan }}^{\circledR} \text { ) }\end{array}$ \\
\hline 292 & 100,0 & $2 \times$ tägliche Hautpflege [19] \\
\hline 292 & 100,0 & $\begin{array}{l}\text { psychologisches Aufnahme- } \\
\text { und Entlassungsgespräch }\end{array}$ \\
\hline 292 & 100,0 & $\begin{array}{l}2 \times \text { wöchentliche Schulung und } \\
\text { ärztlicher Vortrag }\end{array}$ \\
\hline 236 & 80,8 & $\begin{array}{l}\text { Entspannungstraining } \\
\text { (progressive Muskelrelaxation, } \\
\text { Phantasiereisen) }\end{array}$ \\
\hline 147 & 50,3 & $\begin{array}{l}\text { psychologische Einzelgespräche, } \\
\text { Psychotherapie }\end{array}$ \\
\hline
\end{tabular}

reich das psychosoziale Umfeld (sozial, beruflich, schulisch), wie es nur wohnortnah möglich ist.

\section{Dokumentation und Datenerhebung}

Die Ausprägung des psoriatischen Hautbefalls wurde bei Aufnahme und Entlassung mit dem Psoriasisausdehnungs- und Schweregradindex PASI ( = Psoriasis Area and Severity Index) [13] bestimmt. Für jeden Patienten wurde neben der üblichen Krankenakte ein persönlicher Behandlungsbogen geführt, in dem jede Anwendung dokumentiert wurde.

Am Entlassungstag erhielten alle Patienten im Rahmen der Qualitätssicherung in der Rhein-Klinik St. Joseph einen anonym auszufüllenden Fragebogen mit insgesamt 24 Fragen zu Themen wie Zufriedenheit mit Behandlungserfolg, Art der Behandlung, Freundlichkeit der Mitarbeiter, Verpflegung und Unterkunft sowie zu Besonderheiten der wohnortnahen Rehabilitation. Die Rücklaufquote betrug $69,2 \%(\mathrm{n}=202)$. Somit wurden pro Patient bis zu 87 verschiedene Parameter erfasst.

\section{Auswertung und statistische Methoden}

Zur Evaluation wurde mit dem Microsoft-Programm ACCESS ein Datenbanksystem entwickelt, in das die Daten von 292 konsekutiv in der Rhein-Klinik St. Joseph Duisburg in einem Zeitraum von 3 Jahren behandelten Patienten mit Psoriasis vulgaris eingegeben worden sind. Alle statistischen Auswertungen wurden mit dem Programmpaket CSS: Statistica (Fa. StatSoft, USA) durchgeführt.

Die Abheilungsquoten (auch in Tab. 3 und Abb. 2) wurden durch die relative PASI-Reduktion (\%) nach folgender Formel bestimmt: 100 - [100 × (Entlassungs-PASI : Aufnahme-PASI)].
Tab. 2 Klinische Einschätzung des Behandlungsergebnisses durch Arzt und Patient

\begin{tabular}{|llll|}
\hline $\begin{array}{l}\text { Beurteilung } \\
\text { durch den Arzt }\end{array}$ & $\begin{array}{l}\text { Einschätzung } \\
\text { [\%] }\end{array}$ & $\begin{array}{l}\text { Beurteilung } \\
\text { durch den Patient }\end{array}$ & $\begin{array}{l}\text { Einschätzung } \\
\text { [\%] }\end{array}$ \\
\hline komplette Abheilung & 3,1 & sehr gut & 42,9 \\
\hline fast komplette Abheilung & 46,6 & gut & 39,7 \\
\hline deutliche Besserung & 47,9 & zufriedenstellend & 14,7 \\
\hline $\begin{array}{l}\text { geringgradige oder } \\
\text { keine Besserung }\end{array}$ & 1,0 & unbefriedigend & 2,6 \\
\hline & 1,4 (keine & & \\
\hline
\end{tabular}

\section{Ergebnisse}

Bei den 292 konsekutiv behandelten Patienten betrug der Aufnahme-PASI-Score 20,2 $\pm 11,2$ (Median 18) und der EntlassungsPASI-Score 4,5 \pm 4,6 (Median 3,2 Punkte). Der durchschnittliche PASI-Rückgang um 15,7 Punkte (Abb.1) ist statistisch hochsignifikant. Die Abheilungsquote in Bezug auf Schweregrad und Ausdehnung des psoriatischen Befalls erfolgte somit um durchschnittlich 77,7\%, im Median um 82,2\%.

Die Behandlungsergebnisse bei ambulanten und stationären Patienten zeigten keinen signifikanten Unterschied im Rückgang des PASI-Scores (Abb. 1). Im Mittelwert zeigte sich bei den stationären Patienten ein um 0,8 Punkte im PASI-Score (Median 0,45 Punkte) besseres Ergebnis als bei den ambulanten Patienten. Die stationären Patienten verbrachten durchschnittlich 7 Be-

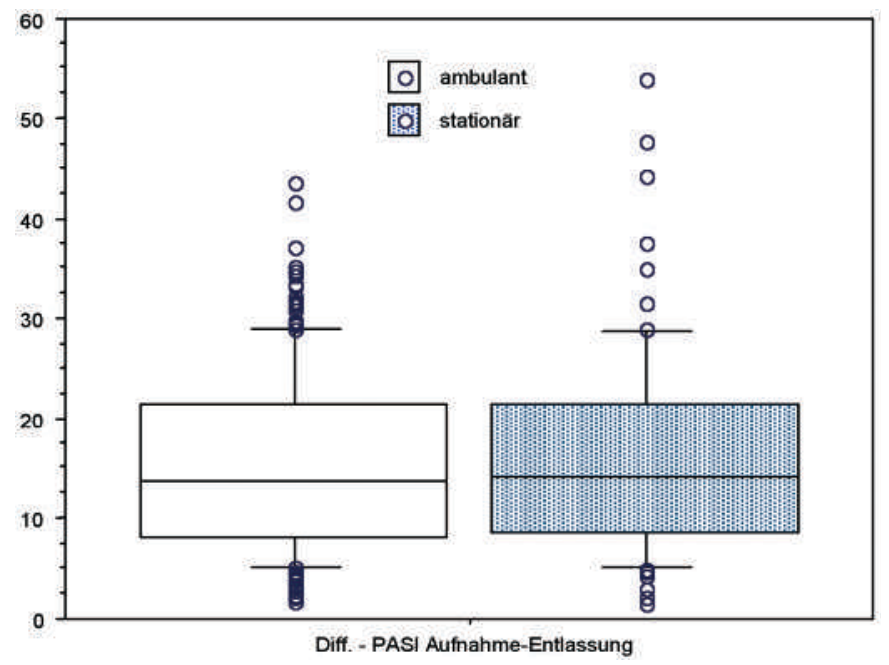

\begin{tabular}{lccc}
\hline & $\begin{array}{l}\Delta \text { PASI Aufn.-Entl., } \\
\text { total }(\mathbf{n}=\mathbf{2 9 2})\end{array}$ & $\begin{array}{l}\Delta \text { PASI Aufn.-Entl., } \\
\text { ambulant }(\boldsymbol{n}=\mathbf{2 0 5})\end{array}$ & $\begin{array}{l}\Delta \text { PASI Aufn.-Entl., } \\
\text { stationär }(\boldsymbol{n}=\mathbf{8 7})\end{array}$ \\
\hline Mittelwert & 15,703 & 15,470 & 16,230 \\
\hline Std.-Abw. & 9,537 & 9,144 & 10,413 \\
\hline SEM & 0,583 & 0,670 & 1,150 \\
\hline Minimum & 1,400 & 1,700 & 1,400 \\
\hline Maximum & 54,000 & 43,600 & 54,000 \\
\hline Median & 13,850 & 13,700 & 14,150 \\
\hline
\end{tabular}

Abb. 1 Reduktion des PASI-Scores in Abhängigkeit von der Art der Behandlung (ambulant/stationär; siehe auch oben stehende Tabelle). 
Tab. 3 Vergleich der primären Rehabilitationserfolge verschiedener Rehabilitationszentren anhand der prozentualen PASI-Score-Reduktion

\begin{tabular}{|c|c|c|c|c|c|c|}
\hline $\begin{array}{l}\text { Publikations- } \\
\text { Jahr }\end{array}$ & Erstautor & $\begin{array}{l}\text { Klinik, } \\
\text { Institution }\end{array}$ & Therapiemethode & Behandlungsdauer & $\begin{array}{l}\text { Patienten- } \\
\text { zahl }\end{array}$ & $\begin{array}{l}\text { mittlere Abhei- } \\
\text { lungsquote [\%] }\end{array}$ \\
\hline 2002 & Ständer [14] & Fachklinik Bad Bentheim & $\begin{array}{l}\text { stationäre Rehabilitation, } \\
\text { Schwerpunkt Balneophoto- } \\
\text { therapie (BPT) }\end{array}$ & $\begin{array}{l}\text { Durchschnittlich } 30 \text { BPT- } \\
\text { Behandlungen pro Patient; } \\
\text { bei angenommenen } 5 \\
\text { Sitzungen pro Woche ca. } 6 \\
\text { Wochen durchschnittliche } \\
\text { Behandlungsdauer }\end{array}$ & 2917 & 92,86 \\
\hline 1997 & Gambichler [16] & Klinikzentrum Bad Sulza & $\begin{array}{l}\text { stationäre Rehabilitation } \\
\text { mit Schwerpunkt } \\
\text { Balneophototherapie }\end{array}$ & 4,4 Wochen & 165 & 82,78 \\
\hline 1997 & Schuh [17] & $\begin{array}{l}\text { Klinik für Dermatologie } \\
\text { und Allergie, Davos }\end{array}$ & $\begin{array}{l}\text { Klimatherapie im Hochgebirge, } \\
\text { wobei die „Heliotherapie“ als } \\
\text { Klimaexposition die Haupt- } \\
\text { komponente darstellte }\end{array}$ & 4 Wochen & 76 & 77,4 \\
\hline 1999 & Lange [18] & $\begin{array}{l}\text { PsoriSol Fachklinik für } \\
\text { Dermatologie und } \\
\text { Allergologie Hersbruck }\end{array}$ & $\begin{array}{l}\text { kombinierte dermatologisch- } \\
\text { verhaltenstherapeutische } \\
\text { stationäre Therapie }\end{array}$ & 25 Tage & 86 & 88,6 \\
\hline 2000 & Petermann [19] & $\begin{array}{l}\text { Fachklinik Sylt für Kinder } \\
\text { und Jugendliche }\end{array}$ & $\begin{array}{l}\text { stationäre Rehabilitation. Der- } \\
\text { matologische Therapie, Klima- } \\
\text { therapie, intensives kognitiv-be- } \\
\text { haviorales Schulungsprogramm }\end{array}$ & 4-6 Wochen & 54 & 50,0 \\
\hline $\begin{array}{l}\text { 2003, [2001], } \\
(1999)\end{array}$ & Buhles [21] & $\begin{array}{l}\text { Asklepios Nordseeklinik } \\
\text { Westerland/Sylt }\end{array}$ & stationäre Rehabilitation & $\begin{array}{l}\text { durchschnittlich } 24 \\
\text { Behandlungstage }\end{array}$ & $\begin{array}{c}123 \\
{[138]} \\
(100)\end{array}$ & $\begin{array}{l}80,0 \\
{[75]} \\
(80)\end{array}$ \\
\hline 2000 & Lemke [22] & $\begin{array}{l}\text { Dermatologisches } \\
\text { Zentrum Buxtehude }\end{array}$ & $\begin{array}{l}\text { wohnortnahe ambulante } \\
\text { Rehabilitation } \\
(5 \times 2,5 \text { h Schulung })\end{array}$ & $\begin{array}{l}20 \text { Behandlungstage, } \\
\text { z. T. auf mehrere Monate } \\
\text { verteilt }\end{array}$ & 27 & 76,9 \\
\hline 2004 & $\begin{array}{l}\text { Kardorff } \\
\text { (eigene } \\
\text { Untersuchung) }\end{array}$ & $\begin{array}{l}\text { Rhein-Klinik St. Joseph } \\
\text { Duisburg, Rehabilitations- } \\
\text { zentrum für Dermatologie, } \\
\text { Allergologie und Umwelt- } \\
\text { medizin }\end{array}$ & $\begin{array}{l}\text { wohnortnahe dermatologische } \\
\text { Rehabilitation, ambulant und } \\
\text { stationär. Kombinierte dermato- } \\
\text { logische und psychologische } \\
\text { Therapie, Schulungen und Ent- } \\
\text { spannungsverfahren. Einbezie- } \\
\text { hung des psychosozialen Umfelds }\end{array}$ & 26 Tage & 292 & 82,2 \\
\hline
\end{tabular}

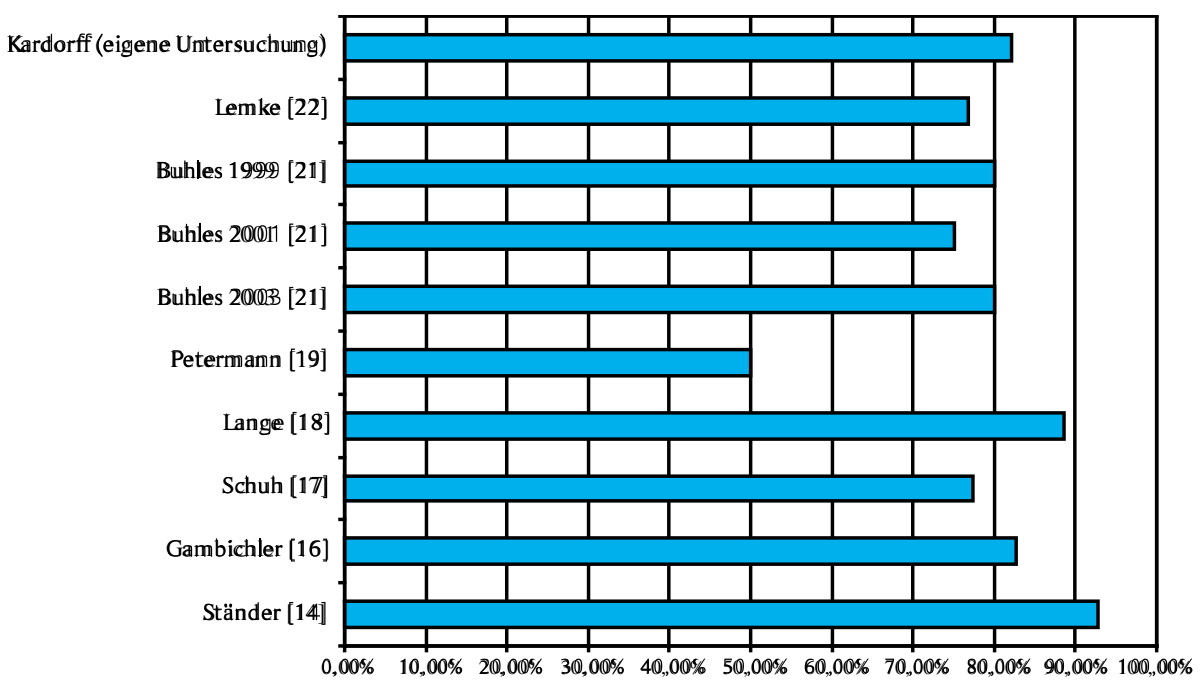

Abb. 2 Vergleich der Abheilungsquoten verschiedener dermatologischer Rehabilitationszentren. handlungstage mehr in der Klinik. Bei annähernd gleicher Behandlungsdauer von ca. 5 Wochen in beiden Gruppen sind diese 7 Tage Differenz durch die Wochenenden zu erklären, die die ambulanten Patienten bei ihren Familien verbrachten, während die stationären Patienten auch an Samstagen und Sonntagen die komplette medizinische Behandlung (Balneophototherapie, Cig-
nolin-Minutentherapie, Hautpflege, etc.) erhielten. Das Auslassen der Therapie in der Rehaklinik an den Wochenenden hatte somit keinen Einfluss auf den Therapieerfolg. Die ambulanten Patienten hatten den Auftrag, die in der Klinik erlernten topischen Behandlungs- und Hautpflegeformen an den Wochenenden direkt in Form eines Praxistests zu Hause fortzuführen. 
Die durchschnittliche Behandlungsdauer betrug 26,3 $\pm 8,5$ Tage ( $\mathrm{n}=292$, Median 26 Tage), ambulant $(\mathrm{n}=205)$ 24,3 \pm 7,5 Tage (Median 25 Tage) und stationär $(\mathrm{n}=87$ ) 31,0 \pm 9,0 Tage (Median 33 Tage). Ambulante Patienten hatten dabei 5 und stationäre Patienten 7 Behandlungstage pro Woche.

Ein Rehabilitationsziel stellt auch die nach Möglichkeit steroidfreie Therapie der Psoriasis dar. Bei Aufnahme erhielten als Vorbehandlung vor Rehabilitationsbeginn 41,8\% der Patienten topische und 6,8\% systemische Steroide zur Psoriasis-Behandlung. Bei Entlassung wurden noch 5 Patienten $(1,7 \%)$ mit topischen Steroiden (im Kopfhautbereich) und kein Patient (0\%) mehr mit systemischen Steroiden behandelt.

Neben der Ermittlung des Entlassungs-PASI-Scores gab der die Abschlussuntersuchung durchführende Arzt auf dem Untersuchungsbogen noch ein deskriptives Urteil über den klinischen Hautzustand der Patienten ab (Tab. 2). Parallel dazu stuften die Patienten auf dem bei Entlassung unter Methodik beschriebenen anonym auszufüllenden Patientenfragebogen ihre Zufriedenheit mit dem persönlichen Behandlungserfolg zwischen sehr gut und unbefriedigend ein. Im Gegensatz zu den Erfahrungen von Ständer et al. [14] zeigten sich hierbei relativ gute Übereinstimmungen zwischen der ärztlichen und der Patientenbeurteilung.

Die für den Aspekt der Wohnortnähe der Rehabilitationsmaßnahme wichtigen Fragen wurden von den rehabilitierten Patienten anonym wie folgt beantwortet:

Den Kontakt zu den eigenen Angehörigen während der Rehamaßnahme erachteten $67,9 \%$ als sehr wichtig und $14,5 \%$ als wichtig (83,4\%), 17,6\% als weniger wichtig oder sogar unwichtig.

Auf die Frage, ob eine Rehabilitationsmaßnahme lieber in größerer Entfernung vom Wohnort hätte stattfinden sollen (Antwortmöglichkeit JA/NEIN), antworteten 12,6\% mit Ja. Der Wunsch nach einer wohnortferneren Rehabilitation stand in keinem statistisch signifikanten Zusammenhang zu der persönlichen Erfolgsbeurteilung.

Die Behandlungskosten für die Kostenträger der Rehabilitation beliefen sich bei ambulanter Behandlung auf 4124,71 DM $(2108,93 €)$ pro Patient und bei stationärer Behandlung auf 6827,48 DM (3490,84€) pro Patient bei annähernd identischem primären Rehabilitationsergebnis. Die stationäre Behandlung erwies sich somit als 65,5\% kostenintensiver. Umgerechnet auf das Behandlungsergebnis kostete jeder Punkt PASI-Score-Reduktion bei ambulanten Patienten 266,63 DM (136,33€) und bei stationären Patienten 420,67 DM (215,09€).

\section{Diskussion}

Noch in der 2. Hälfte der 90er-Jahre des letzten Jahrhunderts gab es erhebliche Bedenken gegenüber der Effektivität der wohnortnahen, „nicht-klimatherapeutischen“ Rehabilitation [15]. Die vorliegende Arbeit stellt das Konzept der wohnortnahen dermatologischen Rehabilitation vor und präsentiert den anhand der PASI-Score-Veränderungen messbaren primären Rehabilitationserfolg. Um zusätzlich aufzuzeigen, welchen Stellenwert die- se therapeutischen Ergebnisse im Ruhrgebiet in Bezug auf den psoriatischen Hautbefall im Vergleich zu traditionellen wohnortfernen Rehabilitationskliniken und einem weiteren Modellprojekt ortsnaher Rehabilitation haben, werden nachfolgend im Text und in der Tab. 3 publizierte Ergebnisse anderer Zentren vorgestellt, die sich auch des PASI zur Evaluation des Therapieerfolgs bedienten:

In der Fachklinik Bad Bentheim wurden zwischen dem 1. 4. 2000 und 1.3.2002 2917 stationär rehabilitierte Psoriasis-Patienten mit einem durchschnittlichen Aufnahme-PASI von 13,83 und einem Entlassungs-PASI von 0,99 im Rahmen eines THESOP II genannten Forschungsprojekts erfasst [14]. Daraus ergibt sich eine Abheilungsquote von 92,86\% (siehe Auswertung und statistische Methoden). Behandlungsschwerpunkt in Bad Bentheim stellt die Balneophototherapie (BPT) dar. Die durchschnittliche Zahl von BPT-Sitzungen pro Patient betrug 30. Bei i.d. R. 5 durchgeführten Behandlungen pro Woche betrug die geschätzte stationäre Verweildauer ca. 6 Wochen. Konkrete Angaben hierzu fehlen leider in der Literaturstelle [14].

Eine Untersuchung aus dem Klinikzentrum Bad Sulza [16] an 165 stationär rehabilitierten Patienten (102 männlich, 63 weiblich, Altersdurchschnitt 44,4 Jahre [17-82 Jahre]) ergab in durchschnittlich 4,4 Therapiewochen eine mittlere Abheilungsquote von $82,8 \%$ bei einem durchschnittlichen Aufnahme-PASI von 15,6 .

Im Rahmen einer Untersuchung der Klinik für Dermatologie und Allergologie in Davos [17] konnte mit der Klimatherapie im Hochgebirge eine Besserung des PASI-Scores von 9,06 auf 2,05 Punkte bei 76 Psoriasis-Patienten beobachtet werden. Dies entspricht einer Abheilungsquote von 77,4\%, wobei eine $43 \%$ ige Besserung bereits in der 1 . Behandlungswoche stattfand. Die „Heliotherapie“ als Klimaexposition stellte dabei die therapeutische Hauptkomponente dar. In einer katamnestischen Untersuchung für Davos wurde eine Rezidivfreiheit über Zeiträume zwischen vier und sieben Monaten bei 27,5\% der Patienten und zwischen acht und zwölf Monaten bei 25,5\% der Patienten festgestellt [17].

86 Psoriasis-Patienten wurden in der PsoriSol-Klinik Hersbruck [18] zwischen September 1997 und März 1998 durchschnittlich 25 Tage stationär mit einer kombinierten dermatologisch-verhaltenstherapeutischen Therapie stationär behandelt. Neben diagnoseorientierten dermatologischen Schulungen wurden Übungen zur Entspannung, zur sozialen Kompetenz, zur Kratzkontrolle sowie psychologische Einzelbetreuung angeboten. Hierbei verbesserte sich der PASI-Score von 23,6 auf 2,7 (Abheilungsquote $88,6 \%)$.

Von der Fachklinik Sylt für Kinder und Jugendliche zusammen mit dem Zentrum für Rehabilitationsforschung der Universität Bremen wurde ein verhaltensmedizinisch orientiertes Patientenschulungsprogramm [19] erarbeitet, welches integrativer Bestandteil der stationären Rehabilitation bei Psoriasis ist. Bei 54 Kindern und Jugendlichen (9-17 Jahre, 23 Jungen, 31 Mädchen), die zwischen Januar 1997 bis Mai 1998 an einer Rehabilitationsmaßnahme teilgenommen haben, kam es im Verlauf der Behandlung zu einem Abfall des PASI von 7,60 auf 3,80 (Abhei- 
lungsquote 50\%). 19 der Kinder und Jugendlichen hatten gegen Ende der Rehabilitationsmaßnahme einen besseren Hautzustand, während sich bei 32 Kindern und Jugendlichen keine Veränderung des Schweregrads zeigte. Bei einem Kind verschlechterte sich der Hautzustand während der Rehabilitationsmaßnahme. Diese vergleichsweise deutlich geringere Besserung des mit dem PASI-Score beurteilten Hautzustands gegenüber den Untersuchungen aus anderen Kliniken (Tab.3, Abb.2) hängt möglicherweise mit der bei Kindern etwas eingeschränkten Palette an Therapiemöglichkeiten der Psoriasis [20] zusammen.

Nach einer persönlichen Mitteilung von Buhles [21] verbesserten sich die durchschnittlichen PASI-Scores der stationären Reha-Patienten der Nordseeklinik Sylt im Jahr 1999 von 15 auf 3, im Jahr 2001 von 12 auf 3 und im Jahr 2003 von 10 auf 2 Punkte bei einer durchschnittlichen Verweildauer von 24 Tagen (Abheilungsquoten $75-80 \%)$.

Bei vergleichender Betrachtung der publizierten PASI-Score-Änderungen im Verlauf rehabilitativer Therapien (Tab.3, Abb. 2) fällt auf, dass die hier vorgestellte wohnortnahe dermatologische Rehabilitation inmitten eines städtischen Ballungsgebiets (Ruhrgebiet) durchaus vergleichbar gute Ergebnisse im Rahmen der Primärevaluation am Ende einer Rehabilitationsmaßnahme bei Psoriasis erbringen kann.

Dies zeigt auch ein anderes, in Buxtehude [22] durchgeführtes Modellprojekt zur wohnortnahen ambulanten dermatologischen Rehabilitation. Es sieht zur Behandlung der Psoriasis 20 durchaus über Monate flexibel zu verteilende Behandlungstage sowie ein modulares Schema mit 5 zweieinhalbstündigen Schulungen u.a. aus den Bereichen Dermatologie, Psychologie und Ökotrophologie vor. Im Rahmen dieses Projekts konnte der PASIScore von anfangs durchschnittlich ca. 13 auf 3 Punkte am Therapieende gebessert werden (Abheilungsquote 76,9\%).

Die hier nebeneinander aufgeführten Ergebnisse sind sicherlich nicht exakt miteinander zu vergleichen, da die Bewertung der Schwere der Psoriasis mittels PASI von verschiedenen Untersuchern unterschiedlich eingesetzt wird und die Bewertung durch verschiedene Autoren oder Institutionen schwankt. Ständer et al. [14] haben daher vorgeschlagen, für Vergleiche nicht die absoluten (PASI-)Werte sondern nur die prozentuale Änderung heranzuziehen (Abb. 2), da davon ausgegangen werden kann, dass Aufnahme- und Entlassungsbefund nach dem gleichen Schema erhoben wurden. Aber auch hierbei ist nur ein tendenzieller Vergleich möglich, da auch der prozentuale Wert z. B. bei einer PASIReduktion um 10 Punkte stark von der Höhe des ermittelten Ausgangswerts abhängt und somit auch keinen objektiven Vergleich ermöglicht.

Zur endgültigen Beurteilung der Qualität der wohnortnahen Rehabilitation wäre eine Langzeitnachbeobachtung der rehabilitierten Patienten mit Nachkontrolle der Entwicklung der PASIWerte wünschenswert. Hierzu gibt es jedoch auch im Bereich der wohnortfernen Rehabilitation nur sehr wenige Daten.

\section{Fazit}

Neben der im Vordergrund stehenden Präsentation der primären Rehabilitationsergebnisse und des wohnortnahen dermatologischen Rehabilitationskonzepts soll die vorliegende Arbeit zum einen aufzeigen, dass auch im Rahmen der direkt ermittelbaren Abheilungsquoten der Psoriasis bei der wohnortnahen Rehabilitation vergleichbar gute Ergebnisse wie bei einer wohnortfernen Rehabilitation bzw. einer Klimatherapie erreicht werden können. Zum anderen sollen auch die hohe Zufriedenheit unseres Patientenkollektivs mit der Rehabilitation in Wohnortnähe sowie die nur wohnortnah vorhandenen Vorteile des ,intrarehabilitativen“ Kontakts zum psychosozialen Umfeld demonstriert werden, wie sie bereits auch für die Rehabilitation von Neurodermitis-Patienten [23] gezeigt werden konnten.

\section{Danksagung}

Hiermit bedanke ich mich bei Herrn Dr. Johannes Kunze (Chefarzt der Hautkliniken Duisburg) und Herrn Prof. Dr. Ulrich R. Hengge (Dermatologische Abteilung der Heinrich-Heine-Universität Düsseldorf [Direktor: Prof. Dr. T. Ruzicka]) für die Überlassung des Themas und die Betreuung der Arbeit.

\section{Literatur}

${ }^{1}$ Richtlinien des Gemeinsamen Bundesausschuss über Leistungen zur medizinischen Rehabilitation nach §92 Abs. 1 Satz 2 Nr. 8 SGB V. Bundesanzeiger Nr. 63 vom 31.3.2004: 6769

${ }^{2}$ Bundesarbeitsgemeinschaft für Rehabilitation Frankfurt (BAR). Rahmenempfehlungen zur ambulanten dermatologischen Rehabilitation vom 22.1.2004

${ }^{3}$ Kardorff B, Kunze J. Präsentation des neuartigen Konzepts „Wohnortnahe Dermatologische Rehabilitation“ am Beispiel einer Patientin mit ausgeprägter Psoriasis vulgaris. Akt Dermatol 1998; 24: 221 - 225

${ }^{4}$ Kardorff B, Schnelle-Parker G, Detering H, Kunze J. Presentation of a new holistic therapy concept for chronic skin diseases in Germany. JEADV 1998; (Supplement 2) 11: 258

${ }^{5}$ Kardorff B. Gesunde Haut. 2. Aufl. Heidelberg: Springer, 2004

${ }^{6}$ Bachert C, Kardorff B, Virchow C. Allergische Erkrankungen in der Praxis. 2. Aufl. Bremen: Uni-Med, 2001

${ }^{7}$ Kardorff B, Kardorff M. Patientenratgeber und kurzes Lexikon der Hautkrankheiten, Venenleiden, allergischen Erkrankungen und kosmetischen Medizin. 1. Aufl. Norderstedt: BOD, 2002

8 Kardorff B, Schnelle-Parker G, Küppers J, Kunze J. Erkrankungsmanagement des Atopischen Ekzems im Kindesalter. Z Hautkr 2001; 76: $77-83$

${ }^{9}$ Internationale Klassifikation der Funktionsfähigkeit, Behinderung und Gesundheit (ICF). Deutsches Institut für medizinische Dokumentation und Information (DIMDI), Stand 24.9. 2002

${ }^{10}$ Runne U, Kunze J. Short-duration ('minutes') therapy with dithranol for psoriasis: a new out-patient regimen. Br J Dermatol 1982; 106: $135-139$

${ }^{11}$ Runne U, Kunze J. Die „Minutentherapie“ der Psoriasis mit Dithranol und ihre Modifikationen. Eine kritische Evaluation von 315 Patienten. Hautarzt 1985; 36 (1): 40-46

${ }^{12}$ Kardorff B, Schnelle-Parker G, Kardorff M, Wahlen M, Hönig d'Orville I, Dorittke P. Erfolgreiche Reduktion des SCORAD-Scores bei Kindern mit atopischem Ekzem im 6-Wochen Vergleich durch Kurzschulung mit einem vereinfachten Hautmodell. JDDG 2003; 1: 451 - 456

${ }^{13}$ Frederickson T, Petterson U. Severe psoriasis - oral therapy with a new retinoid. Dermatologica 1978; 157: 238 - 244

${ }^{14}$ Ständer M, Niederauer HH, Schröpl F. 25 Jahre Balneophototherapie der Psoriasis. Akt Dermatol 2002; 28: 437-442

${ }^{15}$ Strömer K. Dermatologische Rehabilitation: Es gibt keinen adäquaten Ersatz für die Klimatherapie. Dt Ärztebl 1996; 93: A-1952 - 1953 
16 Gambichler T, Böhm S, Poppe J, Schröpl F. Evaluation des primären Rehabilitationserfolges bei Psoriasiskranken. Präv-Rehab 1997; 9: 172 175

17 Schuh A, Topperzer U. Klimatherapie bei Hautkrankheiten Teil II: Behandlung der Psoriasis vulgaris. Münch med Wschr 1997; 139: 315 316

18 Lange S, Zschocke I, Langhardt S, Amon U, Augustin M. Effekte kombinierter therapeutischer Maßnahmen bei Patienten mit Psoriasis und atopischer Dermatitis. Hautarzt 1999; 50: 791 - 797

19 Petermann F, Schmidt S, Warschburger P, Scheewe S, Stachow R. Kognitiv-behaviorales Schulungsprogramm bei Psoriasis. Erste Evaluationsergebnisse. Monatsschr Kinderheilkd 2000; 148: 1104-1112
${ }^{20}$ Zappel K, Sterry W, Blume-Peytavi U. Therapieoptionen der Psoriasis im Kindes- und Jugendalter. JDDG 2004; 2: 329-342

${ }^{21}$ Buhles N. Ergebnisse der stationären Rehabilitation der Asklepios Nordseeklinik Westerland/Sylt. Persönliche Mitteilung, 2004

22 Lemke R, Peter M, Tirre A et al. Training of patients with atopic dermatitis and psoriasis vulgaris in an ambulant neighborhood rehabilitation program: presentation of a pilot project. Dermatol Psychosom 2000; $1: 163$ - 171

${ }^{23}$ Detering H. Ergebnisse der wohnortnahen dermatologischen Rehabilitation bei Patienten mit atopischem Ekzem. Akt Dermatol 2004; 30: $493-499$

\section{Buchbesprechung}

\section{Psychosomatische Dermatologie}

W. Harth, U. Gieler

Heidelberg: Springer, 2005, 310 S., 95 Abb., Geb. 79,95€, ISBN 3-540-24890-0

Zunächst lässt sich über die Autoren sagen, daß sie ausgewiesene Experten auf dem Gebiet der Psychosomatischen Dermatologie sind. Prof. Gieler ist bekannt durch seine Publikationen auf diesem Gebiet und kann als führender Experte der Psychosomatischen Dermatologie in Deutschland bezeichnet werden. Herr Priv.-Doz. Hardt arbeitet ebenfalls seit vielen Jahren auf diesem Gebiet und ist besonders durch Publikation zu Lifestyle-Medikamenten bekannt geworden.

In dem vorliegenden Buch werden die Zusammenhänge zwischen Hauterkrankung, psychischen Faktoren und dem sozialen Umfeld dargestellt, eine Sichtweise, die lange Jahre in der Betrachtung der erkrankten Haut wenig anerkannt und berücksichtigt worden ist.

Das Buch ist in vier Abschnitte gegliedert, die die verschiedenen Facetten und Betrachtungsweisen in dem Spannungsfeld zwischen Haut, Psyche und soziokulturellen Einflussfaktoren darstellen. Die kurz gefasste Einleitung zeigt Definitionen auf, die die Haut als Grenze zwischen Körper und Umfeld verdeutlichen. So können Hauterkrankungen psychische Störungen auslösen (z.B. Entstellungsgefühl), aber auch psychische Erkrankungen zu Hautaffektionen führen (z. B. psychische Exkoriationen).

Im zweiten und größten Abschnitt des Buches werden anhand des derzeitigen Einteilungsprinzips in der psychosomatischen Dermatologie (Dermatosen primär psychischer Genese, Dermatosen mit multifaktorieller Grundlage, deren Verlauf psychischen Einflüssen unterliegen und sekundär psychische Störungen in Folge von schweren oder entstellenden Dermatosen) in der Praxis häufig vorkommende Hauterkrankungen beschrieben.

Dazu gehören Hautartefakte sowie Dermatosen in Folge von Wahnvorstellungen oder Zwangsstörungen, weiterhin somatoforme Störungen (d.h. wiederholte Darbietung körperlicher Symptome in Verbindung mit hartnäckigen Forderungen nach medizinischen Untersuchungen trotz wiederholt negativer Ergebnisse). Zu den multifaktoriellen Dermatosen zählen klassische Hautkrankheiten wie die atopische Dermatitis, Akne-Formen und Psoriasis, bei denen beispielsweise Stress als häufig verschlechternder Einfluss wahrgenommen wird.
Zu den somato-psychischen Störungen zählen die Autoren angeborene und besonders entstellende Dermatosen und deren Folgezustände.

Der dritte Abschnitt geht von speziellen Bereichen in der Dermatologie aus und beschreibt darin psychosomatische Aspekte. So werden beispielsweise in Allergologie, Andrologie, kosmetische Dermatologie oder Photodermatologie auf psychische Faktoren hingewiesen (beispielsweise der Einfluß von Angststörungen). Auch auf den sicher häufig unterschätzten Komplex von Traumatisierungen durch sexuellen Missbrauch wird besonders hingewiesen.

Im vierten Abschnitt werden verschiedene Aspekte aus der dermatologischen Praxis angesprochen. Der interessierte Leser wird hier viele gute Anregungen für den Umgang mit seinen Patienten bekommen: Was ist unter psychosomatischer Grundversorgung zu verstehen, wie funktioniert eine Liaison-Sprechstunde, was kann ich als Dermatologe selbst machen und wo brauche ich eine Zusammenarbeit?

Dem Anhang merkt man an, dass sich die Autoren hier besondere Mühe gegeben haben: Weiterführende Literatur, Kontakt-Links und -Adressen sowie die ICD10-Klassifikation werden in den dermatologischen Praxen sicher gut nutzbar sein.

Besonders wertvoll wird das Buch durch ausgezeichnete Abbildungen, durch erklärende Fallvorstellungen, durch weiterführende Literatur am Ende aller Abschnitte, durch die in der Praxis gut nutzbaren Tipps im vierten Abschnitt des Buches sowie häufige, farblich unterlegte Tabellen und Übersichten.

Aus meiner Sicht ist es allerdings weniger geeignet für „schnell einmal nachschauen“. Die komplexen Zusammenhänge machen es einfach notwendig, dass man sich mit dem Thema näher beschäftigt und sich doch der Mühe unterziehen sollte, die Abschnitte einmal ganz zu lesen.

Das Buch kann besonders empfohlen werden für interessierte Dermatologen, Psychologen, Psychiater, für Pflegeberufe aber auch für Fachärzte für Neurologie, Innere Medizin und Pädiater. Nicht zuletzt sollte es auch den Studierenden der Medizin und Zahnmedizin ans Herz gelegt werden, da hier Probleme angesprochen werden, die oft immer noch im Studium zu kurz kommen.

K.-M. Taube, Halle 